\title{
Use of antidepressants and fracture risk
}

An increased risk of hip or femur fracture for current users of two classes of antidepressants has been observed in a large, case-control study conducted in the Netherlands. The findings also hint that reduced bone strength, rather than increased risk of falling alone, could be a partial reason for this increased risk.

Six thousand, seven hundred and sixty three cases of hip or femur fracture were analyzed, and the mean age of the predominantly female participants was 75 years. Increases in fracture risk were greatest during the initial period of exposure to antidepressants and then declined over time with tricyclic antidepressants, but remained somewhat elevated for selective serotonin reuptake inhibitors. For both classes of antidepressant, the majority of the excess risk dropped to almost baseline values 6-12 months after discontinuation of treatment.

Emerging evidence suggests that inhibition of the serotonin transporter might disturb bone microarchitecture. "In line with this hypothesis, we found the highest risks of hip fracture among users of antidepressants with the highest potential of serotonin transporter inhibition," says Frank de Vries of the University of Utrecht, the study's lead investigator.

de Vries concludes, however, that potential confounding factors, such as smoking, alcohol consumption, reduced exercise and depression itself, were not measured in the case-control study and could have influenced the findings. Nevertheless, "Fracture risk assessment may be indicated among elderly patients who have recently started taking antidepressants," advises de Vries.

Carol Wilson

Original article van den Brand, M. W. M. et al. Use of antidepressants and the risk of fracture of the hip or femur. Osteoporos. Int. doi:10.1007/s00198-009-0849-6 